\title{
Steady-state, elastic-plastic growth of slanted cracks in symmetrically loaded plates
}

Nielsen, Kim Lau; Hutchinson, J. W.

Published in:

International Journal of Impact Engineering

Link to article, DOI:

10.1016/j.jijmpeng.2017.03.015

Publication date:

2017

Document Version

Peer reviewed version

Link back to DTU Orbit

Citation (APA):

Nielsen, K. L., \& Hutchinson, J. W. (2017). Steady-state, elastic-plastic growth of slanted cracks in symmetrically loaded plates. International Journal of Impact Engineering, 108, 286-294.

https://doi.org/10.1016/j.ijimpeng.2017.03.015

\section{General rights}

Copyright and moral rights for the publications made accessible in the public portal are retained by the authors and/or other copyright owners and it is a condition of accessing publications that users recognise and abide by the legal requirements associated with these rights.

- Users may download and print one copy of any publication from the public portal for the purpose of private study or research.

- You may not further distribute the material or use it for any profit-making activity or commercial gain

- You may freely distribute the URL identifying the publication in the public portal

If you believe that this document breaches copyright please contact us providing details, and we will remove access to the work immediately and investigate your claim. 


\title{
Steady-state, elastic-plastic growth of slanted cracks in symmetrically loaded plates
}

\author{
K. L. Nielsen ${ }^{1}$ and J. W. Hutchinson ${ }^{2}$ \\ ${ }^{1}$ Dept. of Mechanical Engineering, Solid Mechanics, Technical University of Denmark, DK- \\ 2800, Lyngby, Denmark \\ ${ }^{2}$ School of Engineering and Applied Sciences, Harvard University, Cambridge, MA, USA
}

\begin{abstract}
Elastic and elastic-plastic results are obtained for a semi-infinite slanted through-crack propagating in a symmetrically loaded plate strip with the aim of providing theoretical background to commonly observed plate tearing behavior. Were it is not for the slant of the crack through the thickness of the plate, the problem would be mode I, but due to the slant the local conditions along the crack front are a combination of mode I and mode III. A threedimensional formulation for steady-state crack propagation is employed to generate distributions of effective stress, stress triaxiality and Lode parameter through the plate in the plastic zone at the crack tip. The distribution of the mode I and mode III stress intensity factors along the crack front are obtained for the elastic problem. The out-of-plane bending constraint imposed on the plate significantly influences the mixed mode behavior along the crack front. The solution is examined for clues as to why propagating slant cracks sometimes undergo a transition and flip about $90^{\circ}$ to propagate with the opposite slant orientation.
\end{abstract}

\section{Introduction}

Under conditions in which a plate is loaded symmetrically with a symmetrically located flat crack, the initial crack advance is also usually symmetric and locally mode I along the crack front. Extensive tearing of metallic sheets and plates has been studied in recent years with the aim of understanding and characterizing extensive failure modes in large plate structures under a wide variety of loading scenarios [1-3]. For plates of ductile metal alloys, a symmetrically loaded cracked plate frequently undergoes a transition as it advances such that its leading edge becomes slanted at approximately $45^{\circ}$ to the plate middle surface [4]. When this happens, conditions along the crack front are no longer pure mode I. In particular, a strong mode III component develops which produces out-of-plane asymmetry and plate bending. Among other 
factors, the transition to the slant crack is due to shear localization that occurs ahead of the crack. More remarkably, when the transition to a slanted crack occurs, it is often observed that the crack front flips back and forth from $+45^{\circ}$ to $-45^{\circ}$ as the crack advances with a regular period that is usually at least several times the plate thickness [5-8]. The mechanics of this flipping mechanism is not understood other than the general realization that it is tied to shear localization occurring in the fracture process. The analysis and results presented in this paper are motivated by slant crack propagation phenomena with the aim of providing some relevant theoretical background. While the flipping process is inherently non-steady, the steady-state solution can give insight as to why flipping might be triggered.

Fig. 1a displays the three-dimensional (3D) plate geometry considered in this paper. The infinite plate strip of thickness $t$ and height $2 H$ has a semi-infinite crack with slant angle $\beta_{S}$ which intersects the plate mid-surface at the plate symmetry line $\left(x_{2}=0, x_{3}=0\right)$. The tractions on the lateral faces of the strip plate are zero as are the tractions on the crack faces. The boundary conditions along the bottom and top edges of the plate are taken to be

$$
\begin{aligned}
& \sigma_{12}\left(x_{1},-H, x_{3}\right)=\sigma_{13}\left(x_{1},-H, x_{3}\right)=0, \quad u_{2}\left(x_{1},-H, x_{3}\right)=-\Delta, u_{3}\left(x_{1},-H, 0\right)=0 \\
& \sigma_{12}\left(x_{1}, H, x_{3}\right)=\sigma_{13}\left(x_{1}, H, x_{3}\right)=0, u_{2}\left(x_{1}, H, x_{3}\right)=\Delta, u_{3}\left(x_{1}, H, 0\right)=0
\end{aligned}
$$

For the numerical finite element model introduced below, the length of the plate strip, $2 L$, is taken to be finite but sufficiently long to simulate steady-state propagation conditions with the crack tip located at the halfway point of the length.

The geometry and boundary conditions on the top and bottom edges are consistent with the existence of a steady-state solution. The condition $u_{3}\left(x_{1}, \pm H, 0\right)=0$ imposed at the center of the plate on the bottom and top edges restrains the middle surface plate from out-of-plane displacement along this line. It will produce a concentrated line force/per length in the $x_{3}-$ direction along the centerline of the top and bottom edges when out-of-plane bending occurs. This is an important constraint, which together with the thickness to half-height ratio $t / H$, plays a central role influencing mode III conditions at the crack front. In combination, the boundary conditions effectively clamp the plate against out-of-plane rotation on the bottom and top edges while at the same time allowing local shearing on the edges. Thus, for an uncracked plate of 
initially uniform isotropic material, as well as for the plate strip far ahead of the crack, these boundary conditions admit a uniform uniaxial tensile stress state $\left(\sigma_{22}>0\right.$, other components zero) with associated vertical strain $\varepsilon_{22}=\Delta / H$. Far behind the crack tip the stresses completely are relaxed in the elastic problem.

After the 3D steady-state formulation and its numerical implementation is introduced, the first problems addressed will be those for a linear elastic material. Results for the throughthickness distribution of the mode I and mode III elastic stress intensity factors for the slanted crack will be presented which illustrate the role of the out-of-plane bending. The last sections of the paper present the results for the steadily growing elastic-plastic slant crack, having first made contact with mode I results for the flat crack $\left(\beta_{S}=0\right)$ obtained by Sobotka and Dodds [9].

\section{3D steady-state formulation and numerical implementation}

\subsection{Constitutive model (J2-flow material)}

J2-flow plasticity theory is adopted as a material model in the present analysis with the current stress field determined from the incremental constitutive law: $\dot{\sigma}_{i j}=L_{i j k l} \dot{\varepsilon}_{k l}$. Here, $L_{i j k l}$ is the elastic stiffness tensor during elastic unloading $\left(\sigma_{e}<\sigma_{e}^{\max }\right.$ or $\left.\dot{\sigma}_{e}<0\right)$, or the instantaneous moduli based on the von Mises surface during plastic yielding $\left(\sigma_{e}=\sigma_{e}^{\max }\right.$ and $\left.\dot{\sigma}_{e}>0\right)$. The Mises effective stress is $\sigma_{e}^{2}=3 / 2 s_{i j} s_{i j}$ with $s_{i j}$ as the Cauchy stress deviator. A power hardening law is chosen for the stress-strain response in uni-axial tension such that;

$$
\mathcal{E}=\left\{\begin{array}{ccc}
\frac{\sigma_{e}}{E} & \text { for } & \sigma_{e} \leq \sigma_{Y} \\
\frac{\sigma_{Y}}{E}\left(\frac{\sigma_{e}}{\sigma_{Y}}\right)^{1 / N} & \text { for } & \sigma_{e}>\sigma_{Y}
\end{array}\right.
$$

where $E$ is Young's modulus, $\sigma_{y}$ is the initial yield stress, and $N$ is the power hardening exponent. Poisson ratio is $v$. A small strain model formulation with the total strain field determined from the current displacements, $\varepsilon_{i j}=\left(u_{i, j}+u_{j, i}\right) / 2$, is used. The strain is 
decomposed into an elastic, $\varepsilon_{i j}^{E}$, and plastic, $\varepsilon_{i j}^{p}$, contributions such that $\varepsilon_{i j}=\varepsilon_{i j}^{E}+\varepsilon_{i j}^{p}$. Thus, the plastic strain field can be determined through the elastic stress-strain relation as: $\varepsilon_{i j}^{p}=\varepsilon_{i j}-M_{i j k l}^{E} \sigma_{k l}$ (exploited in the steady-state procedure below), once the current stress field is known. Here, with $M_{i j k l}^{E}$ being the isotropic elastic compliance tensor. The material parameters used in the present study are $\sigma_{Y} / E=0.004, v=0.3$ and $N=0.1$ matching those used in Sobotka and Dodds [9].

\subsection{Steady-state formulation and numerical procedure}

Dean and Hutchinson [10] and Parks et al. [11] define steady-state for a crack, propagating at a constant velocity, as the condition for which the stress and strain fields surrounding the advancing crack tip remain unchanged to an observer moving with the tip. Thus, any time rate of change, $\dot{f}$, can be related to the spatial derivative through the crack tip velocity, $\dot{a}$, along the $x_{1}$-direction, so that; $\dot{f}=-\dot{a} \frac{\partial f}{\partial x_{1}}$. An incremental quantity, in a given material point, $x_{i}^{*}$, can thereby be evaluated by streamline integration along the negative $x_{1}$-direction, which starts well in front (upstream, $x_{1}=x_{10}>>0, x_{2}=x_{2}^{*}$, and $x_{3}=x_{3}^{*}$ ) of the active plastic zone and ends at the point of interest; $x_{i}=x_{i}^{*}$. In the steady-state, the deformation history at $x_{i}^{*}$ is contained in all upstream material points.

In the following, a steady-state solution is approximated by using a numerical finite element procedure similar to that of [10-14]. The conventional principle of virtual work for quasi-static steady-state problems reads

$$
\int_{V} \delta \varepsilon_{i j} L_{i j k l}^{E} \varepsilon_{k l} d V=\int_{S} \delta u_{i} T_{i} d S+\int_{V} \delta \varepsilon_{i j} L_{i j k l}^{E} \varepsilon_{k l}^{p} d V
$$

where $\varepsilon_{i j}$ is the total strain, $\varepsilon_{i j}^{p}$ is the plastic strain tensor, $L_{i j k l}^{E}$ is the elastic stiffness tensor, $u_{i}$ is the displacement vector, and $T_{i}$ is the surface traction vector. The numerical framework is based on Eq. (4), which in 3D is discretized by 20-node isoparametric solid elements using full Gauss 
quadrature for the integration. The basis of the numerical steady-state procedure is summarized below (iterated quantities are indicated by the superscript " $n "$ ):

1) Based on the plastic strains from the previous iteration, $\varepsilon_{i j}^{p(n-1)}$, solve Eq. (4) to obtain the current displacement field, $u_{i}^{(n)}$.

2) Compute the total strain, $\varepsilon_{i j}^{(n)}$, from the current displacement field $u_{i}^{(n)}$.

3) Determine the stress field outside of the steady-state domain: $\sigma_{i j}^{(n)}=L_{i j k l}^{E} \varepsilon_{k l}^{(n)}$, and inside of the steady-state domain:

$$
\sigma_{i j}^{(n)}=\int_{x_{10}}^{x_{1}^{*}} \frac{\partial \sigma_{i j}^{(n)}}{\partial x_{1}} d x_{1} \quad, \text { with; } \quad \frac{\partial \sigma_{i j}^{(n)}}{\partial x_{1}}=L_{i j k l} \frac{\partial \varepsilon_{i j}^{(n)}}{\partial x_{1}}
$$

4) Compute the plastic strain field inside the steady-state domain using the elastic relationship: $\varepsilon_{i j}^{p}=\varepsilon_{i j}-M_{i j k l}^{E} \sigma_{k l}$.

5) Repeat Steps 1 through 4 until satisfactory convergence is achieved. Convergence in both the displacement field and the stress field is here considered.

The iterative steady-state procedure is carried out by taking appropriate differences between Gauss points on the same streamline, by assuming a linear variation in the total strain field between the neighboring integration points. This enables easy division of the interval into smaller subincrements (never less than 100). For initiation of the procedure, the algorithm is fed the elastic solution of the corresponding boundary value problem. Thus, $\varepsilon_{i j}^{p(0)}=0$ in the first iteration $(n=1)$.

To model the plate geometry in Fig. 1a only the upper half of the plate is considered, while appropriate rotational symmetry has been enforced to account for a slant propagating crack. In comparison to modeling the full plate, this approach has proven efficient and with no effect on the results presented. For the elastic-plastic simulations, the through-thickness direction is discretized by 20 elements (graduate finer towards the free surfaces), and with a fine discretization in the $x_{2} x_{3}$-plane near the crack tip covering a domain of 0.25-0.5 times the 
reference value, $\left(K_{R} / \sigma_{Y}\right)^{2}$, that approximately scales the plastic zone height. The fine discretized near tip domain of 24 by 24 elements is refined towards the slant crack surface. The total mesh consists of 70640 20-node 3D elements and 298591 nodes (see Fig. 1b). A similar mesh is used for the elastic calculations, but here with the elements push toward the crack tip such that a very fine resolution of the crack face displacement is obtained.

The current three-dimensional steady-state model has been validated against a recent study on crack growth in thin sheet metals by Sobotka and Dodds [9], as well as corresponding 2D models $[14,15]$, which are known to agree well with the predictions made in earlier papers $[10,12,16]$.

\section{Stress intensity factors for a slant crack in an elastic plate strip}

The geometry for the elastic slant crack problems is that in Fig. 1a. This problem is studied first to provide basic elastic results for the strip plate problem. Although the iteration process required for steady-state elastic-plastic problems is not needed for the linear elastic problems, the same formulation without iteration can be employed to generate the elastic results. The elastic problems also serve to guide the choice of finite element mesh. The plate material is assumed to be uniform and isotropic with Young's modulus $E$ and Poisson's ratio $v$.

The 3D $J$-integral is a useful tool for the elastic problems, especially as a check on the accuracy of the numerical results. Let $S$ be any surface within the plate (see Fig. 1a) that surrounds the crack front with outward pointing unit normal $\mathbf{n}$. The surface $S$ intersects the lateral traction-free faces of the plate on both sides and intersects both traction-free crack faces behind the crack front. With $W=\sigma_{i j} \varepsilon_{i j} / 2$ as the elastic energy density of the plate material, the 3D $J$-integral provides the energy release rate $\bar{G}$ averaged over the crack front as

$$
\bar{G} t=\int_{S}\left(W n_{1}-T_{i} u_{i, 1}\right) d S, \text { with } T_{i}=\sigma_{i j} n_{j}
$$

Using techniques similar to those employed originally by Rice [17], one can readily show that the integral is surface-independent in the sense that the integral is the same for all such $S$ [18]. The fact that the crack faces and the lateral plate surfaces are traction-free with no $x_{1}$-component of $\mathbf{n}$ is essential to this property. 
Consider a surface $S$ which far ahead of the crack front is planar with normal $\mathbf{n}=(1,0,0)$, extending from the bottom edge to the top edge, and far behind the crack front is also planar with normal $\mathbf{n}=(-1,0,0)$ extending from the bottom and top edges to the crack faces. Between these two planar surfaces, take the surfaces of $S$ to coincide with the bottom and top planar edges. The integrand of Eq. (5) vanishes for all the planar surfaces making up this $S$ except for the plane far ahead of the crack. Far ahead of the crack tip the stress state is uniaxial tension, $\sigma_{\infty}=E \Delta / H$, leading to the result from (3): $\bar{G}=E \Delta^{2} / H=\sigma_{\infty}^{2} H / E$. Next, shrink $S$ down to a circular cylindrical surface connecting the two lateral faces of the plate with the straight crack front as the center of the cylinder. At every interior point along the crack front the singular fields are a combination of the singular fields for plane strain mode I and for mode III. The stress intensity factors of these fields, $K_{I}(s)$ and $K_{I I I}(s)$, vary with position $s$ along the front. By shrinking the cylinder $S$ down to zero radius and asserting surface-independence, one obtains from (5)

$$
\bar{G}=\frac{1}{t} \int_{-t / 2 \cos \beta_{S}}^{t / 2 \cos \beta_{S}}\left\{\frac{1-v^{2}}{E} K_{I}(s)^{2}+\frac{1+v}{E} K_{I I I}(s)^{2}\right\} d s
$$

where $s$ is position along the front measured from the plate mid-plane. The 3D corner singularity [19] where the crack front intersects the lateral faces of the plate does not alter this result which depends on the distribution of the intensity factors through the interior of the crack front.

In the numerical simulations, the elastic stress intensity factor distribution is evaluated by fitting the local finite element displacements near the crack front to the displacement fields associated with the plane strain mode I and mode III singularity fields. No effort was made to characterize or capture the amplitude of the corner singularity. More sophisticated techniques for evaluating the intensity factors could be used, but this method proved satisfactory for present purposes where the main objective for the elastic problem is to understand the role of the out-ofplane bending constraint on the mode I and mode III contributions.

Fig. 2 presents the distribution of the mode I stress intensity factors over the range of plate thickness to half-height, $0.01 \leq t / H \leq 0.5$, for the flat crack $\left(\beta_{S}=0\right)$. The problem is 
mode I and the intensity factor is normalized by $K_{R}=\sqrt{\bar{G} E}=\sigma_{\infty} \sqrt{H}$. The distribution is symmetric with respect to the mid-surface of the plate. Note that the variation of $K_{I} / K_{R}$ through the thickness is very small, not more than about $3 \%$. The strip with the smallest height, $t / H=0.5$, flattens the distribution. Our numerical method is not expected to accurately capture the details of the distributions very near the crack faces where the corner singularity influences the distribution. Also shown is the plot of the ratio $\bar{G}_{I} / \bar{G}$ as a function of $t / H$ where the average $\bar{G}_{I}$ is evaluated numerically using the distribution $K_{I}$ in (6). The close agreement between $\bar{G}_{I}$ and $\bar{G}$ is an indicator of the accuracy of the numerical method.

Fig. 3 presents the distribution of the mode I and III stress intensity factors over the same range of thickness to plate half-height for the slant crack $\left(\beta_{S}=45^{\circ}\right)$. The intensity factors are again normalized by $K_{R}=\sigma_{\infty} \sqrt{H}$. Each distribution is symmetric with respect to the midsurface of the plate; $K_{I}(s)$ is maximum at the mid-surface while $K_{I I I}(s)$ is maximum at the faces. The strip plate with the smallest height considered, $t / H=0.5$, produces the largest mode III stress intensity factor and component average $\bar{G}_{I I I}$. This implies that increasing the strip height, thereby relaxing the out-of-plane bending constraint, lowers the mode III contribution in the elastic case.

As already emphasized, the numerical method is not expected to accurately capture the details of the distributions very near the crack faces where the corner singularity influences the local distribution. Plots of the individual mode I and mode III contributions to the energy release rate on the right hand side of Eq. (6), labeled as $\bar{G}_{I}$ and $\bar{G}_{I I I}$, respectively, are shown in the lower part of Fig. 3. The sum of these two components constitutes the numerical evaluation of $\bar{G}$ based on the integral in Eq. (4). Thus, results for the sum of $\bar{G}_{I}$ and $\bar{G}_{I I I}$ in Fig. 3 demonstrate again that surface-independence is accurately met except for the smallest halfheights where it begins to be eroded. The accompanying out-of-plane shearing displacement, $\Delta_{3}=u_{3}\left(-r, 0^{+}, 0\right)-u_{3}\left(-r, 0^{-}, 0\right)$, normalized by the prescribed strip separation, $\Delta=\sigma_{\infty} H / E$, is presented as a function of $-x_{1} / H$ in Fig. 4. The out-of-plane contribution to $K_{I I I}$ would be 
positive if $\Delta_{3}>0$ and vice versa. Relaxation of the mode III component by out-of-plane bending is consistent with the negative sign of $\Delta_{3}$.

Variations of the two contributions to the average energy release rate, $\bar{G}_{I}$ and $\bar{G}_{I I I}$, and their sum are shown in Fig. 5 as a function of the slant crack angle from $\beta_{S}=0^{\circ}$ to $\beta_{S}=45^{\circ}$. These have been computed using the numerical technique just described. The sum of the computed rates is again compared to the prescribed value, $\bar{G}=\sigma_{\infty}^{2} H / E$ revealing the same level of accuracy noted for the $45^{\circ}$ slant crack. These results reinforce the earlier assertion that the out-of-plane bending of the strips with the greatest height provides the largest relaxation of the mode III contribution.

The following elementary approximate calculation for the two energy release rate contributions provides further insight. Assume that at each point along the crack front the only displacement contribution of order $\sqrt{r}$ to the relative displacement of the crack faces is $u_{2}$, where $r$ is the perpendicular distance from the front. That is, ignore the asymmetry of the slant crack with respect to the plate mid-surface $x_{3}=0$ by ignoring the out-of-plane contribution of $u_{3}$ to the crack face displacement such that the only contribution has the form $u_{2}=c(s) \sqrt{r}$. With

$$
\left(u_{2}^{\prime}, u_{3}^{\prime}\right)=c(s) \sqrt{r}\left(\cos \beta_{s}, \sin \beta_{s}\right)
$$

as the associated components of displacement in local axes aligned with the crack front, one can easily show by enforcing (6) that

$$
\left(\bar{G}_{I}, \bar{G}_{I I I}\right)=\frac{\bar{G}}{\left(\cos \beta_{S}\right)^{2}+(1-v)\left(\sin \beta_{S}\right)^{2}}\left(\left(\cos \beta_{S}\right)^{2},(1-v)\left(\sin \beta_{S}\right)^{2}\right)
$$

The dependency on the slant crack angle is shown in Fig. 6 for several values of $v$. The curves for $t / H=0.01$ and $v=0.3$ from Fig. 5 are also included in the figure. While this approximation provides no information on the position-dependence of the stress intensity factors along the crack front, it does clearly capture the trend of their contributions to the average energy release rate. 


\section{Steady-state, elastic-plastic results for the flat crack $\beta_{S}=0^{\circ}$}

The numerical method described in Section 2 has been applied to the mode I flat crack with $\beta_{S}=0^{\circ}$ primarily to provide a base of comparison with the slant crack and to make contact with the prior 3D results for this problem given by Sobotka and Dodds [9]. The dimensionless load parameter used here is the same as that employed in [9]: with $K_{R}=\sigma_{\infty} \sqrt{H}$ as the measure of applied load, the load parameter is $K_{R} /\left(\sigma_{Y} \sqrt{t}\right)$. The plastic zone (where the effective stress satisfies $\sigma_{e} \geq \sigma_{Y}$ ) is shown in Fig. 7 for two values of the dimensionless load, the lower level for a case with the plastic zone diameter less than one-half the plate thickness and the higher level having a plastic zone about ten times the thickness. These zones are in close accord with the corresponding zones for steady-state growth presented in [9]. Note that the zone is fairly uniform in shape through most of the thickness with a slight decrease in size just inside the free surfaces for the smaller of the two zones with $K_{R} /\left(\sigma_{Y} \sqrt{t}\right)=1$, similar to the zone presented for this loading in [9]. Moreover, the shape of this smaller zone is similar to the $3 \mathrm{D}$ zone reported for monotonic loading in small scale yielding by [21], apart from the existence of the reloading wake for the growing crack.

It is worth recording that the shape of the smaller of the two zones in Fig. 7 is quite different from the depiction of such zones presented in a number of texts on fracture. The standard depiction has the zone significantly expanding in size as it approaches the free surfaces. To our knowledge, the textbook depictions are not based on numerical simulations but rather are qualitative based on the idea that a plane strain zone exists in the center region of the plate (which is correct) and a plane stress-like zone exists at the surfaces (which is not correct). While the state of stress on the surface is plane stress, plasticity at the surface is nevertheless subject to the 3D constraint of the surrounding elastic region. Only when the plastic zone becomes considerably larger than the plate thickness, as in the case of the larger zone in Fig. 7, can the outer region of the zone be characterized as plane stress.

Sobotka and Dodds [9] present an array of detailed results on the stress and strain distribution at the tip of a $3 \mathrm{D}$ crack making contact with $2 \mathrm{D}$ results for mode I for both plane strain and plane stress. The focus in this paper will be on distributions near the crack tip of the three invariants of 
stress. Figs. 8 and 9 give contour plots of the distribution of the Mises effective stress, the triaxiality, $T=\sigma_{m} / \sigma_{e}$, with $\sigma_{m}=\sigma_{k k} / 3$ as the mean stress, and the Lode parameter,

$$
L=\frac{2 \sigma_{I I}-\sigma_{I}-\sigma_{I I I}}{\sigma_{I}-\sigma_{I I I}}
$$

where $\left(\sigma_{I} \geq \sigma_{I I} \geq \sigma_{I I}\right)$ are the principal stresses. These plots are for values at the free surface $\left(x_{3}=t / 2\right)$ and at the mid-surface $\left(x_{3}=0\right)$. The loading in Fig. 8 is $K_{R} /\left(\sigma_{Y} \sqrt{t}\right)=1$, while that in Fig. 9 is $K_{R} /\left(\sigma_{Y} \sqrt{t}\right)=5$, in both cases for $t / H=0.01$.

The significant difference between the effective stress contours in Fig. 8 and 9 are consistent with the remarks made above. When the plastic zone is small compared to the plate thickness, the effective stress distribution is much closer to the plane strain distribution than the plane stress distribution even at the plate surface. Only when the zone is large compared with the thickness, as in Fig. 9, is the distribution similar to the 2D plane stress distribution, at least at distances from the tip greater than $t$.

To interpret the distribution on the Lode parameter in Figs. 8 and 9, one should note that $L=0$ corresponds to a shearing stress state (i.e., a shear stress in some axes orientation plus hydrostatic tension or compression), while $L= \pm 1$ corresponds to axisymmetric stressing (i.e., $L=-1$ corresponds uniaxial stress in some orientation plus hydrostatic tension or compression while $L=1$ corresponds equi-biaxial stress in some orientation plus hydrostatic tension or compression). For the smaller of the two plastic zones in Fig. 8e,f, the Lode parameter directly ahead of the crack tip across the central region is approximately $L \cong 0.5$ and then becomes $L \cong 1$ at the surface. Across the entire crack front, $L=0$ along rays approximately $\pm 35^{\circ}$ to the crack plane. For the smaller zone, the triaxiality is largest in a relatively broad region ahead of the crack tip both in the interior and at the surface. It is also significant that the triaxiality is almost as large along rays at $\pm 45^{\circ}$ to the crack plane as directly ahead of the tip. For the larger plastic zone, in Fig. 9e,f, $L \cong 0$ in a fairly broad region ahead of the tip. The Lode parameter ahead of the crack tip, $L \cong 0$, is consistent with a state of plane strain tension $\left(\sigma_{11}=\sigma_{22} / 2, \sigma_{33}=0\right)$ with triaxality, $T=1 / \sqrt{3}$, which is very close to the computed values $T \cong 0.6$. For a material 
susceptible to the void mechanism of failure, these conditions are favorable for out-of-plane shear failure on one or other of the planes at $\pm 45^{\circ}$ to the plate mid-surface.

\section{Steady-state elastic-plastic results for the slant crack with $\beta_{S}=45^{\circ}$}

Three-dimensional views of the active plastic zone at four levels of the normalized load parameter, $K_{R} /\left(\sigma_{Y} \sqrt{t}\right)$, are shown in Fig. 10 for the 45 -degree slant crack. First and foremost one sees that the size of the plastic zone roughly scales with $\left(K_{R} / \sigma_{Y}\right)^{2}$ such that for the lowest load intensity $\left(K_{R} /\left(\sigma_{Y} \sqrt{t}\right)=1\right)$, the diameter of the plastic zone is roughly $0.4 t$ and about $8 t$ when $K_{R} /\left(\sigma_{Y} \sqrt{t}\right)=5$. In addition, a thin active plastic zone is observed close to the crack surfaces in the wake behind the advancing crack tip where a small amount of reverse plastic flow occurs. All-in-all, this behavior is similar to that for the corresponding flat crack. However, the slanting asymmetry develops in the active plastic zone due to the mode III contribution discussed previously. In particular, the asymmetry is obvious for the lowest values of the normalized load parameter $\left(K_{R} /\left(\sigma_{Y} \sqrt{t}\right)=1\right.$ or 2 , see Fig. 10a-b), while it diminishes somewhat when the load parameter is increased (see Fig. 10c-d). Thus, when the plastic zone size is smaller than the plate thickness a significant slanting asymmetry arises, whereas when the plastic zone is much larger than the plate thickness there is relatively little through-thickness variation. Similar behavior is also observed in the contour plots of the three stress invariants presented in Figs. 11-12 for the larger value of normalized load. For, $K_{R} /\left(\sigma_{Y} \sqrt{t}\right)=5$ the free surface contours are hard to distinguish from the contours plotted at $x_{3}=t / 3$ (compare Fig. 12a,c,e to Fig. 12b,d,f). The effective stress, stress triaxiality and Lode parameter all remain roughly constant through thickness except very near the crack tip which Fig. 12 does not resolve. By contrast, for the lowest normalized load, $K_{R} /\left(\sigma_{Y} \sqrt{t}\right)=1$ in Fig. 11, a fairly significant through-thickness variation exists. At the free surface (Fig. 11a,c,e), a clear downward pointing trend is seen in all parameters of interest. To help interpret these figures, note that the free surface contours are the view of the surface seen from the left in Fig. 10a, and similarly for the distributions at $x_{3}=t / 3$. The effective stress contour has a kidney shape, but with one part situated downward along the $x_{2}$-axis and the other lobe along the growth direction of the $x_{1}$-axis with a slight downward bend. 
In a similar fashion, the slant crack tip emits a ray of increasing stress triaxiality in a roughly $45^{\circ}$ angle, and the Lode parameter is zero (shearing stress states) along two rays that meet up near the crack tip, one being roughly in the crack growth direction, and one angled downwards. The downward trend in the various fields, and the asymmetry, gradually weakens as one moves along the crack front into the plate, as is evident from Fig. 11b,d,f.

For a ductile plate that fails by the void growth mechanism, the downward pointing trend of the contours near the free surface suggests that the crack is encouraged to alter its direction of propagation. It is well known that void growth develops most rapidly in high triaxiality regions undergoing plastic deformation, and these are the conditions in the downward pointing region near the slant crack tip. The fact that the Lode parameter is nearly zero in this downward pointing region as well further favors shear localization. These results nicely support a recent Xray tomography study presented by Nielsen and Gundlach [21], where crack tip flipping is observed to start as shear-lips where the slant crack intersects the free surface. Upon further loading, the initiated flip crack grows and overtakes the parent slant crack until the crack face flips completely to the opposite 45-degree orientation. Moreover, the finding that the throughthickness variation within the active plastic zone heavily depends on the normalized load parameter also suggests that the flipping mechanism (driven by the asymmetry) may be restricted to a specific range of plate thicknesses dependent on the material yield stress. When the plastic zone becomes large compared to the sheet thickness, the driving force for the flipping becomes small due to the loss of the downward trends in the distributions noted in connection with Fig. 12. In the early experimental study of the phenomenon by El-Naaman and Nielsen [7], thin sheets of different thicknesses were tested without clear evidence of flipping. Only when thicker plates ( $t \cong 4 \mathrm{~mm}$ for plates of moderate strength steel) were tested was reproducible flipping observed.

The near tip out-of-plane deflection for the elastic-plastic case is presented in Fig. 13 for thickness to plate half-height $t / H=0.01$, representing the plate strip with the least out-of-plane bending constraint. A clear shift in the direction of the out-of-plane plate deflection is observed as the load intensity increases, with the purely elastic case presenting a negative deflection (as discussed in the previous section) and the most heavily loaded $\left(K_{R} /\left(\sigma_{Y} \sqrt{t}\right)=5\right)$ elastic-plastic case showing a strongly positive deflection. When the plastic zone becomes larger than the plate thickness, plastic shear ahead of the crack front overrides the tendency seen for the elastic slant 
crack for the out-of-plane deflection to reduce the mode III contribution. The significant positive crack face mode III displacement just behind the crack tip for the two higher loadings is evident in Fig. 13. This shearing explains why the Lode parameter is zero on the extended plane ahead of the crack (see Figs. 12e,f).

A competition between the far-field elastic response and the near tip plasticity is at play here. One sees this trend with the reduction of the load intensity in Fig. 13. In fact, for the lowest load intensity (i.e., $K_{R} /\left(\sigma_{Y} \sqrt{t}\right)=1$, when the size of the plastic zone compares to the plate thickness), the plate is found to deflect in the negative out-of-plane direction near to the crack tip while becoming positive direction some distance downstream from the crack tip. For these plate strips with substantial height (e.g., $t / H=0.01$ ), the load level $K_{R} /\left(\sigma_{Y} \sqrt{t}\right)=2$ appears to roughly mark the transition above which the plate no longer displays a negative near-tip out-ofplane deflection. Interestingly, in the plate tearing experiments by Simonsen and Törnqvist [6] and by El-Naaman and Nielsen [7], where an edge crack in large steel and aluminum plates is observed to flip repeatedly, the normalized load parameter $K_{R} /\left(\sigma_{Y} \sqrt{t}\right)$ is estimated to be about 1.9 for two sets of the steel plates and 1.3 for the aluminum plates.

Fig. 14 shows the role of the out-of-plane bending constraint via variations in the plate thickness to half-height, $t / H$, for fixed load intensity, $K_{R} /\left(\sigma_{Y} \sqrt{t}\right)=1$. For low values of $t / H$ , corresponding to a limited constraint on the deflection, the plate displays negative out-of-plane deflection close to the crack tip, while the deflection becomes positive further downstream. By contrast, as the constraint on the out-of-plane deflection intensifies with larger $t / H, \Delta_{3}$ remains negative and attains a negative plateau. Increasing the out-of-plane bending constraint of the plate reduces the positive mode III plastic shearing contribution.

\section{Conclusions}

The 3D formulation for steady-state growth of slant cracks in symmetrically loaded elasticplastic plate strips has been used to gain insight into the propagation behavior of slant cracks. The steady-state formulation permits generation of behavior in the plastic zone for a crack that has undergone extensive crack advance without the necessity of computing the transient growth to reach steady-state. New results for the elastic problem are a byproduct of the study in the form of distributions of mode I and mode III stress intensity factors along the crack front. The 
study also makes contact with the earlier 3D steady-state study for elastic-plastic behavior for mode I flat cracks by Sobotka and Dodds [9]. The primary results are for the $45^{\circ}$ slant crack in the form of distributions of the three stress invariants within the plastic zone and the role of the out-of-plane bending constraint on these stress distributions. The results are examined with a focus on identifying clues for the tendency of slant cracks in certain plate tearing tests to flip back and forth between $\pm 45^{\circ}$. While crack tip flipping is a non-steady process, the steady-state solution provides insights into the onset of the advance of a flipping crack.

The numerical results reveal that for the plate strips with the least out-of-plane bending resistance $(t / H=0.01)$ there is a range of loading as measured by $K_{R} /\left(\sigma_{Y} \sqrt{t}\right)$ in which conditions may be favorable to crack tip flipping. In the acute angle corner where the slant crack intersects the plate surface the stress distribution appears to be consistent with initiation of a reoriented shear crack in a the flipping direction. These conditions involve relatively high effective stress and triaxiality with a Lode parameter, $L \cong 0$, associated with shearing states. If $K_{R} /\left(\sigma_{Y} \sqrt{t}\right)$ is much greater than 2 , the plastic zone begins to become so large compared to the thickness that these favorable conditions disappear. Estimates for three sets of tests for which periodic flip-flop was observed gave $K_{R} /\left(\sigma_{Y} \sqrt{t}\right) \cong 1.9$ for two sets of moderate strength steel plates and $K_{R} /\left(\sigma_{Y} \sqrt{t}\right) \cong 1.3$ for one set of aluminum plates. There was no clear evidence of flipping for a set of thinner aluminum plates with higher values of $K_{R} /\left(\sigma_{Y} \sqrt{t}\right)$.

The experimental plates just mentioned had heights comparable to the highest strip plates in the theoretical study and thus can be considered to have relatively low resistance to outof-plane bending. The numerical simulations for the strip plates show that constraining the outof-plane deformation by considering strips with less height, i.e., larger $t / H$, reduces the crack tip contribution due to plastic shearing ahead of the slant crack. While the Young's modulus has not been varied in our numerical studies, it is expected that increasing the elastic modulus will have a constraining effect on the out-of-plane bending displacement similar to the effect of decreasing the height of the strips. In experiments with aluminum and steel plates the increase of modulus associated with the steel plates was the apparent reason for the larger flipping frequency of the steel plates relative to the aluminum plates (see Fig. 1 in [7]). Thus, the authors speculate that the plate height becomes an influential factor in the flipping process due to its influence on the out-of-plane constraint. 
It is important to bear in mind that a small strain formulation has been employed in the present paper which is not capable of capturing necking within the plastic zone ahead of the crack tip. Thus, the present study does not shed light on any influence sheet necking might have on distribution of the stresses in the plastic zone and any role this might have in the flipping mechanism.

\section{Acknowledgement}

K.L.N. has been financially supported by the Villum Foundation Young Investigator Programme in the project "A New Phenomenon Yet to be Resolved in ductile PLATE tearing”, grant VKR023451. J.W.H. has been partially supported by a grant from the US Office of Naval Research (ONR: N00014-16-1-2552).

\section{References}

[1] Aune V, Fagerholt E, Hauge KO, Langseth M, Borvik T. Experimental study on the response of thin aluminum and steel plates subjected to airblast loading . Int. J. Impact Eng. 2016; 90:106-121.

[2] Gruben G, Morin D, Langseth M, Hopperstad OS. Strain localization and ductile fracture in advanced high-strength steel sheets. Eur. J. Mech. A/Solids 2017; 61: 315-329.

[3] Gruben G, Langseth M, Fagerholt E, Hopperstad OS. Low-velocity impact on high-strength steel sheets: An experimental and numerical study. Int. J. Impact Eng. 2016; 88: 153-171.

[4] Hickey WF, Ravi-Chandar K. Transition from flat to slant fracture in ductile materials. Springer International Publisher, Ch. 9, 215-235.

[5] Pardoen T, Hachez F, Marchioni B, Blyth PH, Atkins AG. Mode I fracture of sheet metal. J Mech Phys Solids 2004;53:423-452.

[6] Simonsen BC, Törnqvist R. Experimental and numerical modeling of ductile crack propagation in large scale shell structures. Marine Struct. 2004;17:1-27.

[7] El-Naaman SA, Nielsen KL. Observations on mode I ductile tearing in sheet metals. Eur. J. Mech. A/Solids 2013;42:54-62. 
[8] Felter CF, Nielsen KL. Assisted crack tip flipping under mode I thin sheet tearing ", Eur. J. Mech. A/Solids 2017 (accepted for publication).

[9] Sobotka JC, Dodds RH. Steady crack growth in a thin, ductile plate under small-scale yielding condtions: Three-dimensional modeling. Eng. Fract. Mech. 2011; 78: 343-363.

[10] Dean RH, Hutchinson JW. Quasi-static steady crack growth in small-scale yielding. In: Fracture mechanics: twelfth conference, ASTM STP700. American Society for Testing and Materials; 1980: 383-405.

[11] Parks DM, Lam PS, McMeeking RM. Some effects of inelastic constitutive models on crack tip fields in steady quasistatic growth. Advances in fracture research. In: Francois D editor. Fifth international conference on fracture, Cannes, France. 1981;5; 2607-2614.

[12] Wei Y, Hutchinson JW. Steady-state crack growth and work of fracture for solids characterized by strain gradient plasticity. J. Mech. Phys. Solids 1997;45:1253-1273.

[13] Niordson CF. Analysis of steady-state ductile crack growth along a laser weld. Int J Fract 2001;111:53-69.

[14] Nielsen KL, Niordson CF. Rate sensitivity of mixed mode interface toughness of dissimilar metallic materials: studied at steady state. Int. J. Solids and Struct. 2012; 49:576-583.

[15] Nielsen KL, Niordson CF, Hutchinson JW. Strain gradient effects on steady state crack growth in rate-sensitive materials. Eng. Fract. Mech. 2012;96:61-71.

[16] Suo Z, Shih CF, Varias AG. A theory for cleavage cracking in the presence of plastic flow. Acta Metall. Mater. 1993;41:1551-7.

[17] Rice JR. A path-independent integral and the approximate analysis of strain concentration by notches and cracks. J. Appl. Mech. 1968; 35: 379-386.

[18] Nakamura T, Shih CF, Freund LB. Nonlinear fracture mechanic: Vol. I-Time-dependent fracture. ASTM STP 995, American Society for Testing and Materials, Philadelphia; 1989: 217232.

[19] Bazant ZP, Estenssoro LF. Surface singularity and crack propagation. Int. J. Solids Struct. 1979; 15: 405-426.

[20] Nakamura T, Parks DM. Three-dimensional crack front fields in a thin ductile plate. J. Mech. Phys. Solids 1999; 38: 787-812.

[21] Nielsen KL, Grundlach C. Crack tip flipping under mode I tearing: Investigated by X-ray tomography. 2016; submitted for publication. 


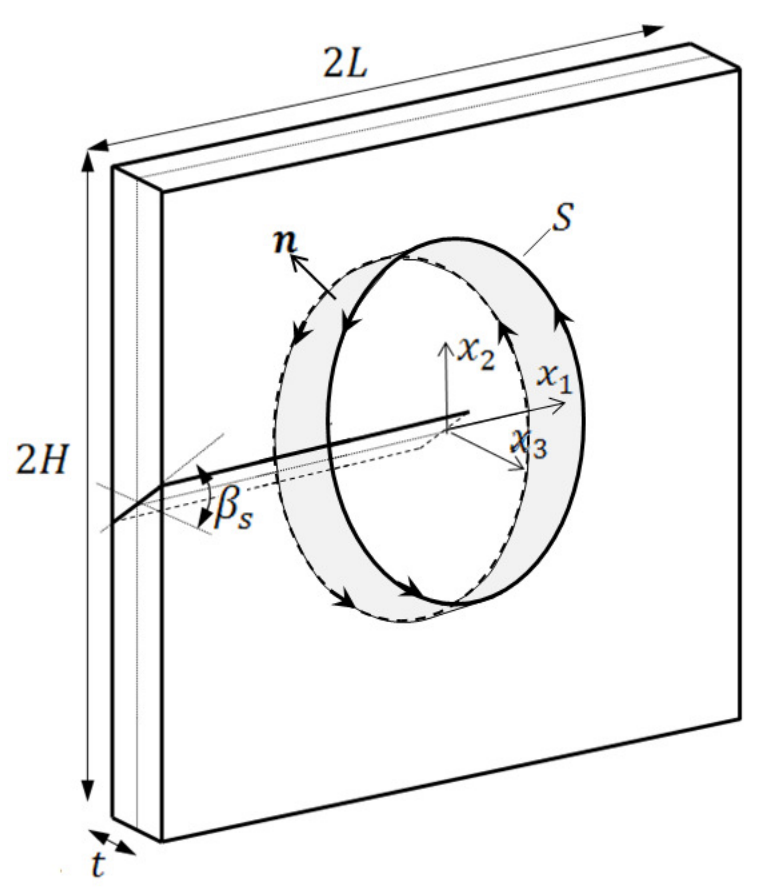

(a)

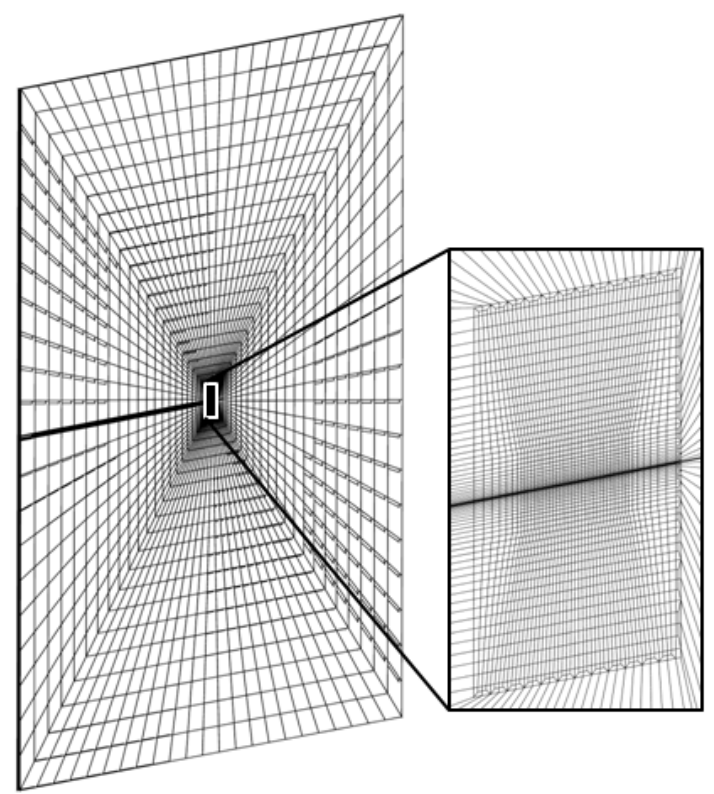

(b)

Fig. 1: a) 3D geometry of the problem at hand and a representative surface $S$ associated with evaluation of the average energy release rate in Eq. (6). Only the upper half of the domain with proper rotational boundary conditions to mimic the full plate is considered in the numerical simulations, b) representative mesh employed in the elastic-plastic simulations (here for $K_{R} /$ $\left(\sigma_{Y} \sqrt{t}\right)=1, t / H=0.01$, and $\left.\beta_{S}=45^{\circ}\right)$. 


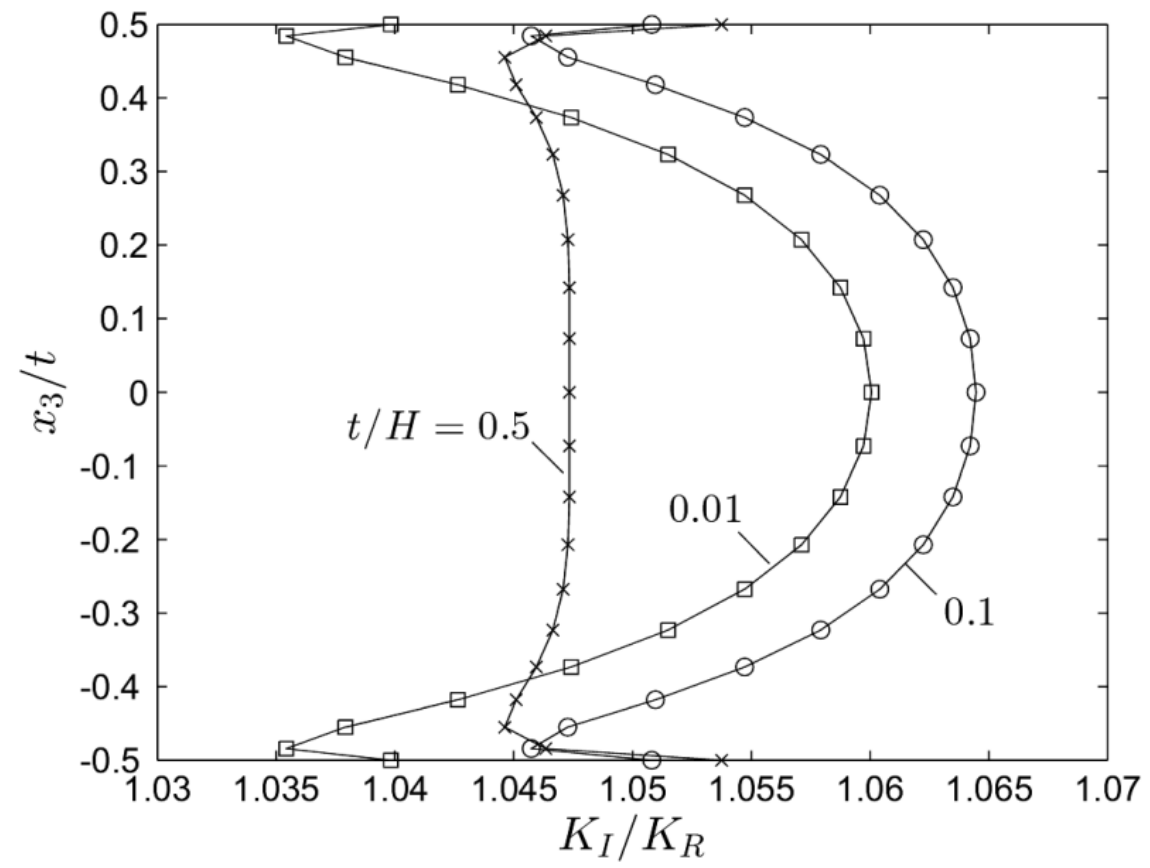

(a)

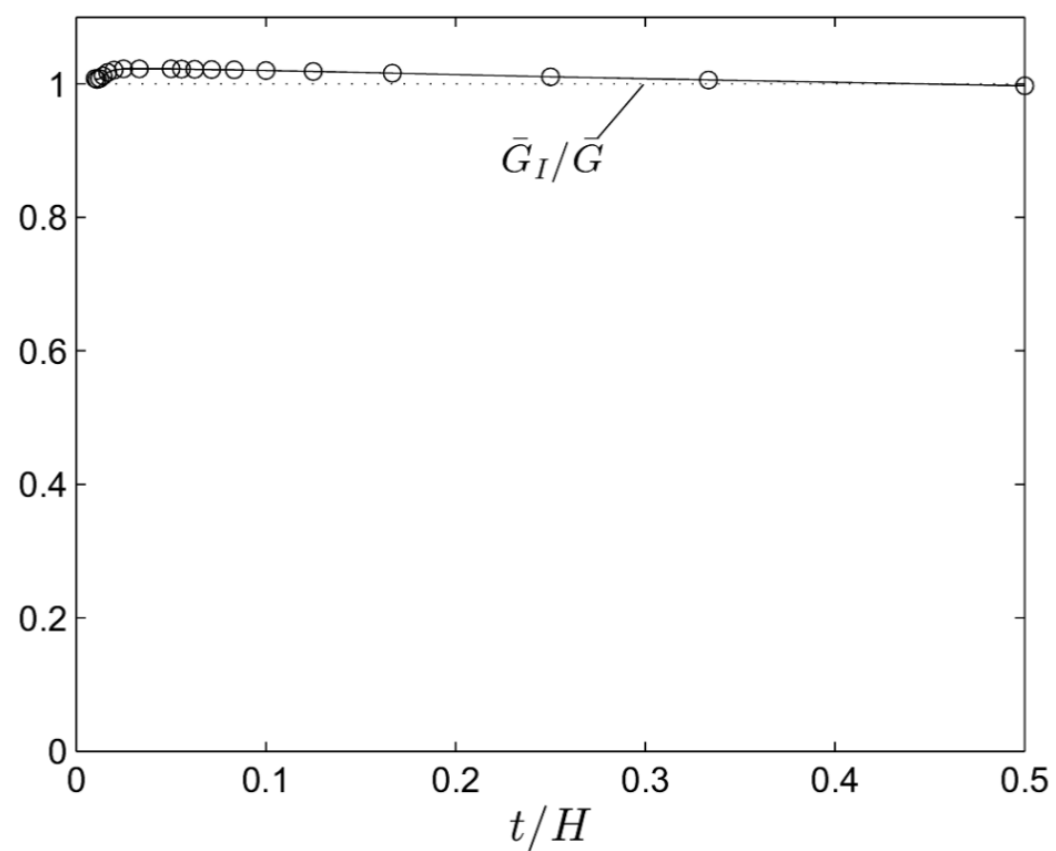

(b)

Fig. 2: Flat crack, $\beta_{s}=0$, for elastic problem (Poisson's ratio, $v=0.3$ ), showing a) the throughthickness distribution of mode I stress intensity factor, and b) the numerical evaluation of the average energy release rate using Eq. (4) normalized by the imposed average energy release rate: $\bar{G}=E \Delta^{2} / H$. 


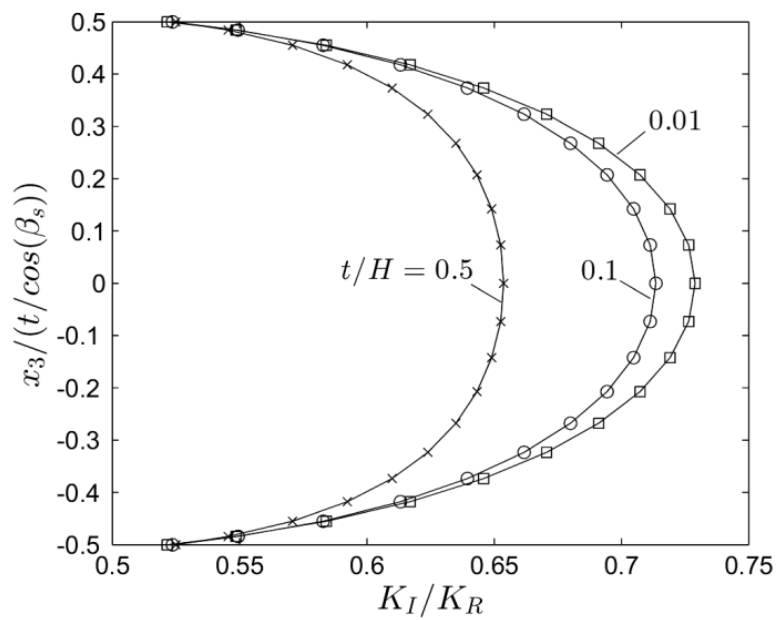

(a)

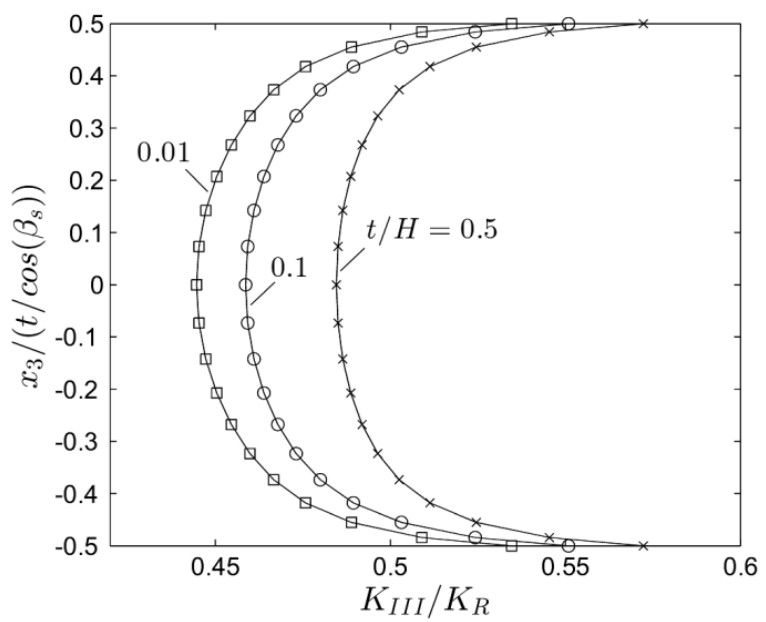

(b)

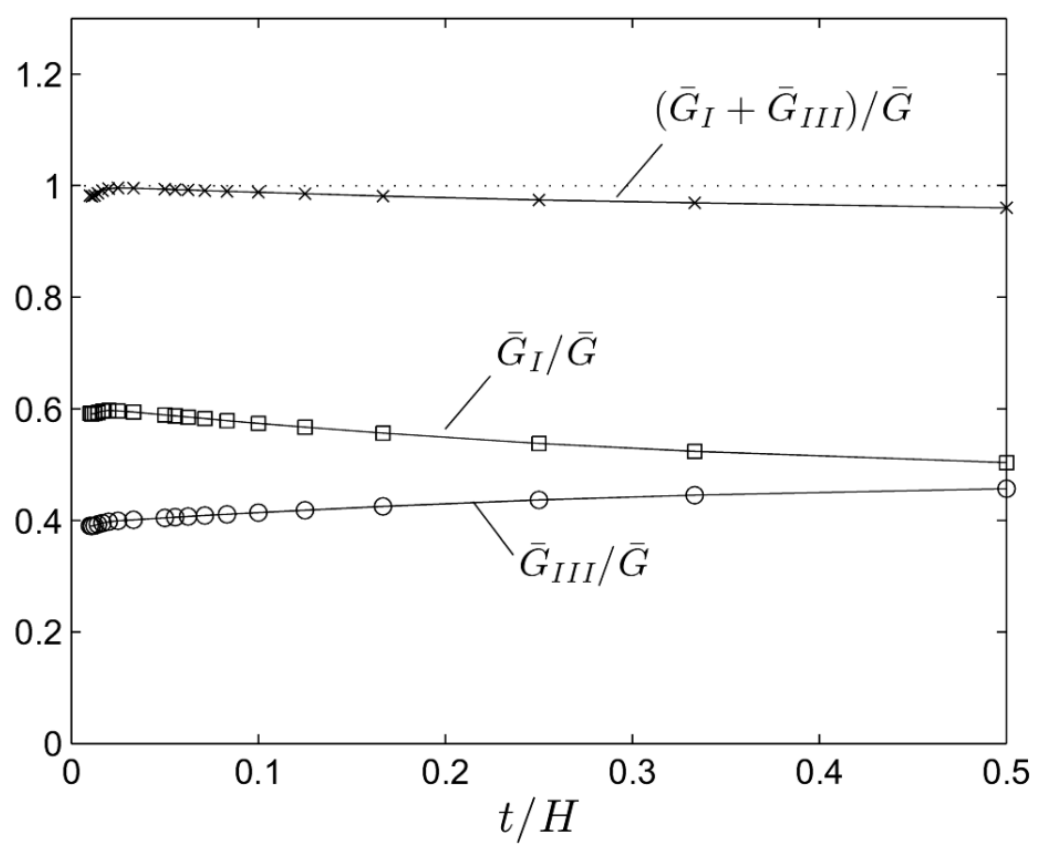

(c)

Fig. 3: Slant crack, $\beta_{s}=45^{\circ}$, for elastic problem (Poisson's ratio, $v=0.3$ ), showing a) the through-thickness distribution of mode I stress intensity factor, and b) the through-thickness distribution of mode III stress intensity factor, and c) the numerical evaluation of the average components of the energy release. 


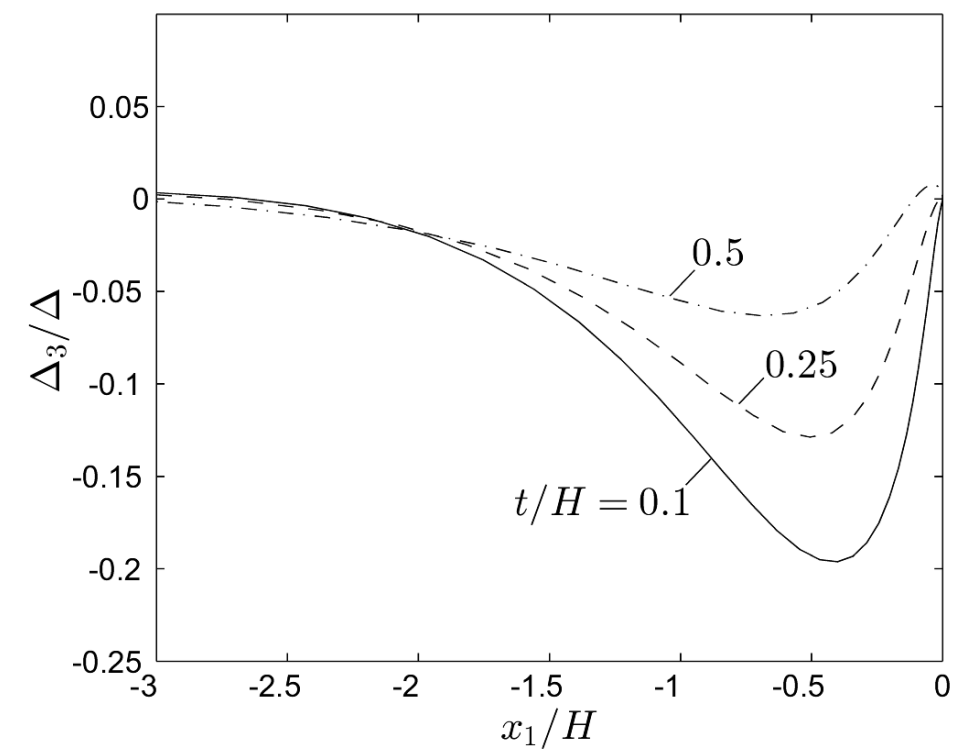

Fig. 4: Out-of-plane deflection at mid-thickness for the elastic problem $(v=0.3)$.

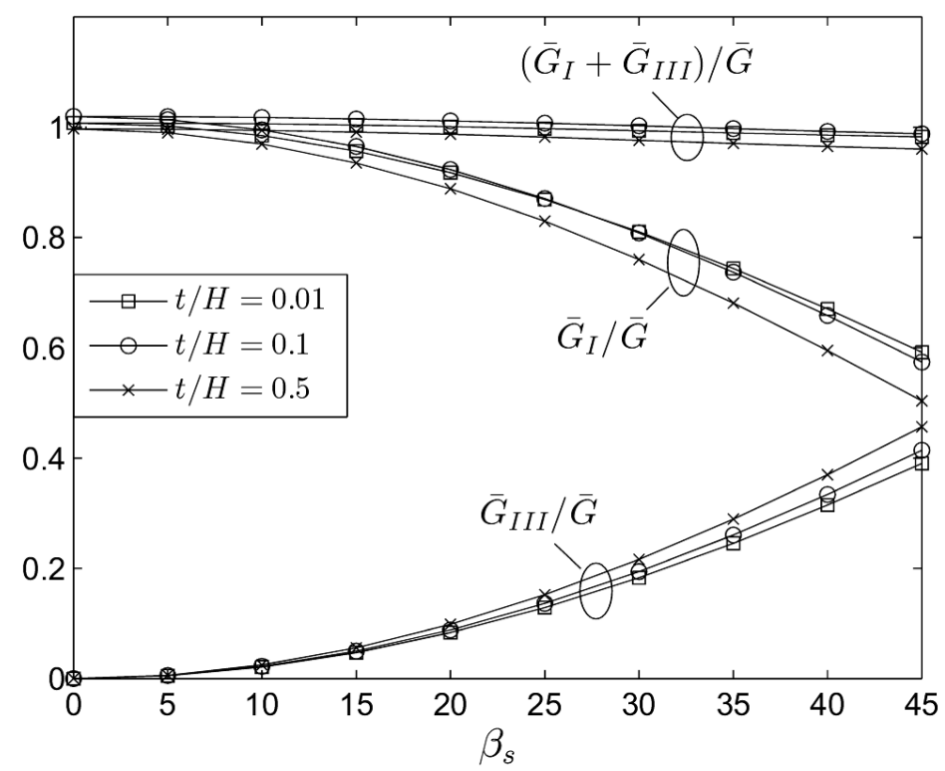

Fig. 5: Numerical results for the elastic problem $(v=0.3)$ for the average mode I and III components of the energy release rate as dependent on the slant angle, $\beta_{s}$ and $t / H$. 


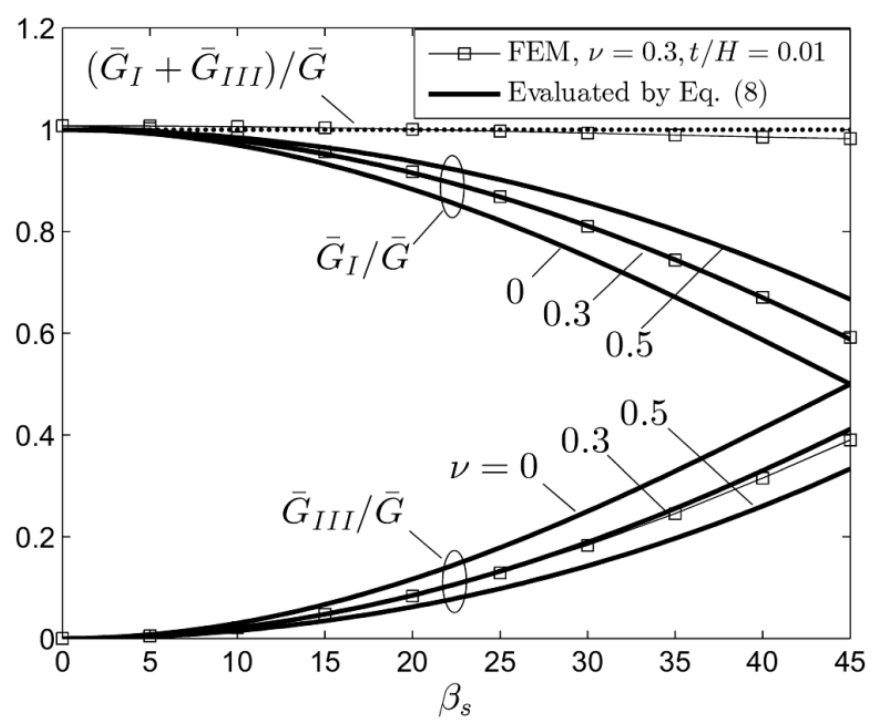

Fig. 6: Approximate results of Eq. (8) for average energy release rate components based only on the assumption of no out-of-plane crack face displacement contribution to the stress intensities compared with the numerical results for $t / H=0.01$ and $v=0.3$.

a)

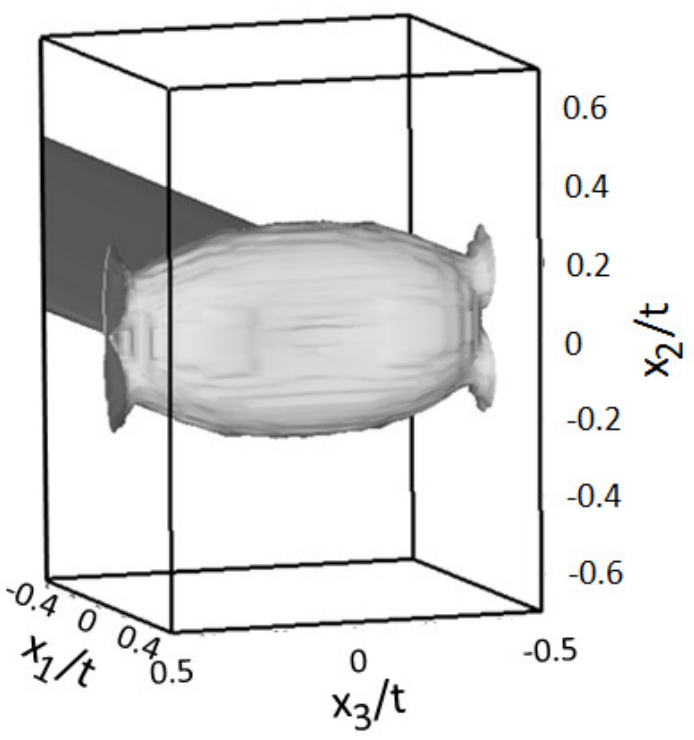

b)

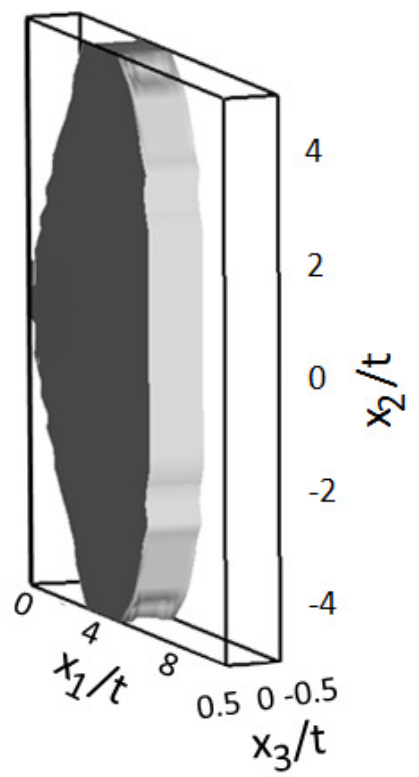

Fig. 7: Active plastic zone for a flat crack propagating at steady-state. Here with the loading corresponding to a) $K_{R} /\left(\sigma_{Y} \sqrt{t}\right)=1$, and b) $K_{R} /\left(\sigma_{Y} \sqrt{t}\right)=5$. Axes are normalized by the sheet thickness 

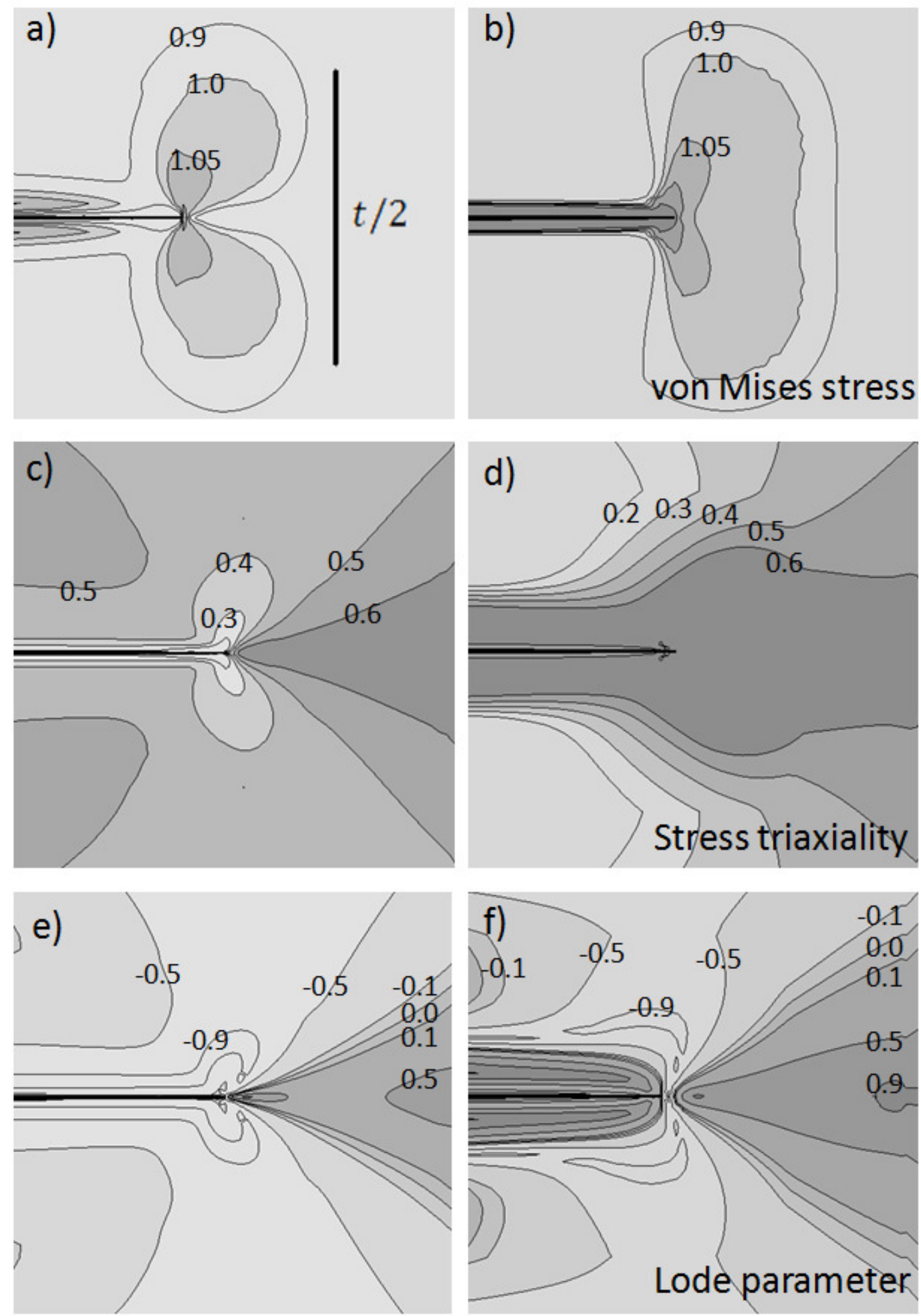

Fig. 8: Contour plot at $x_{3}=t / 2$ (free surface, plots; a, c, e) and at $x_{3}=0$ (plots; b, d, f). Here, showing; a-b) normalized von Mises stress $\left(\sigma_{e} / \sigma_{y}\right)$, c-d) Stress triaxiality, and e-f) Lode parameter, $\left(K_{R} /\left(\sigma_{Y} \sqrt{t}\right)=1, t / H=0.01\right.$, and $\left.\beta_{S}=0\right)$. 

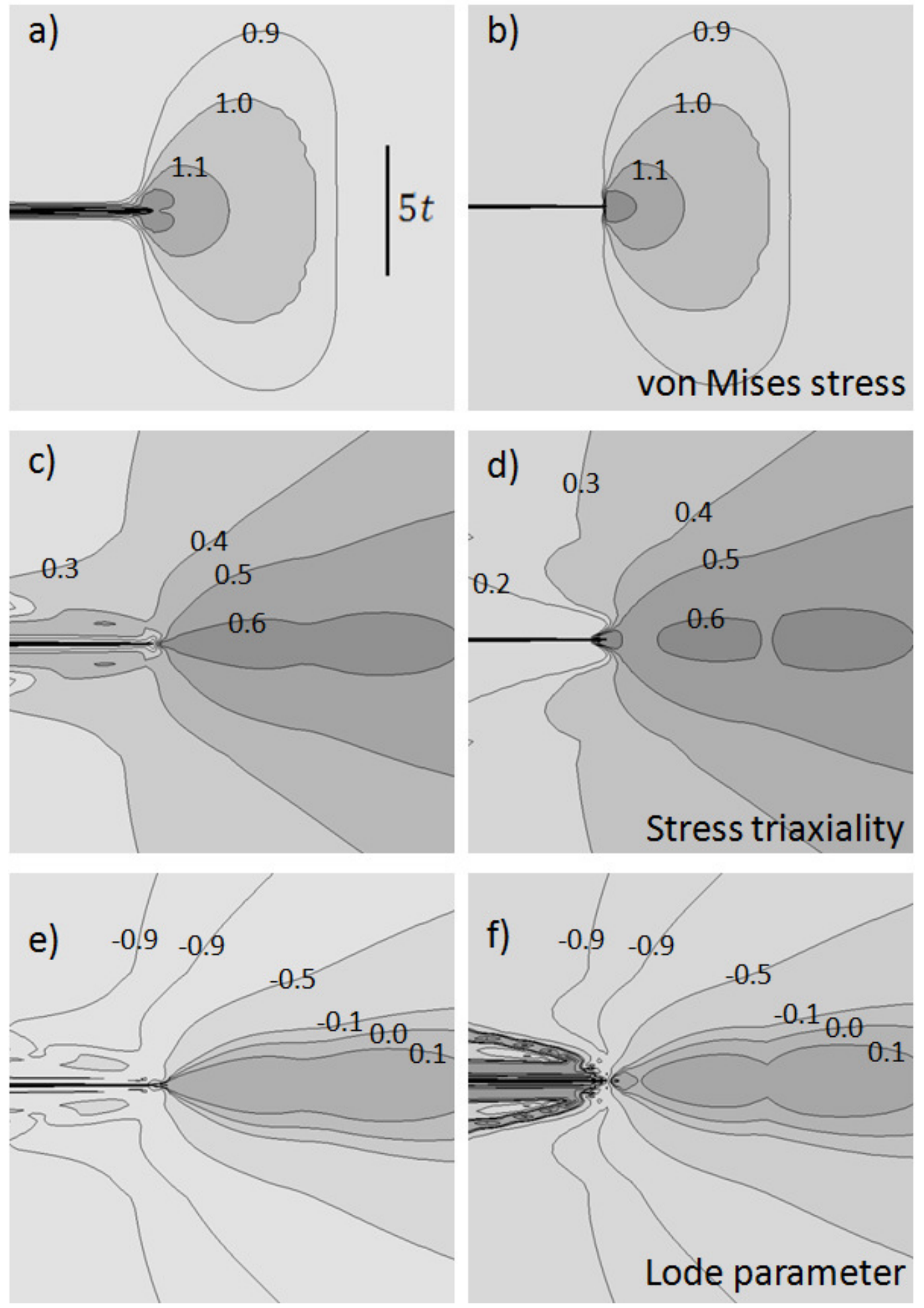

Fig. 9: Contour plot at $x_{3}=t / 2$ (free surface, plots; a, c, e) and at $x_{3}=0$ (plots; b, d, f). Here, showing; a-b) normalized von Mises stress $\left(\sigma_{e} / \sigma_{y}\right)$, c-d) Stress triaxiality, and e-f) Lode parameter, $\left(K_{R} /\left(\sigma_{Y} \sqrt{t}\right)=5, t / H=0.01\right.$, and $\left.\beta_{S}=0\right)$. 
a)

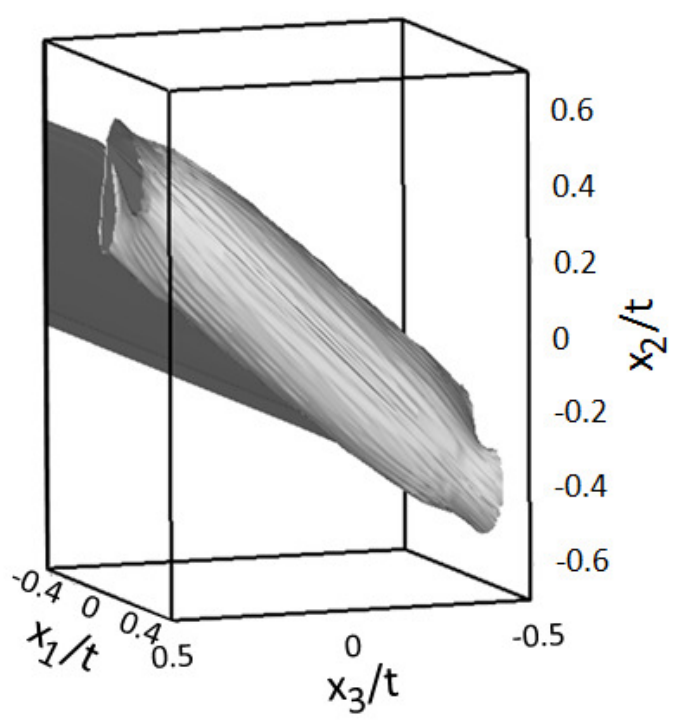

c)

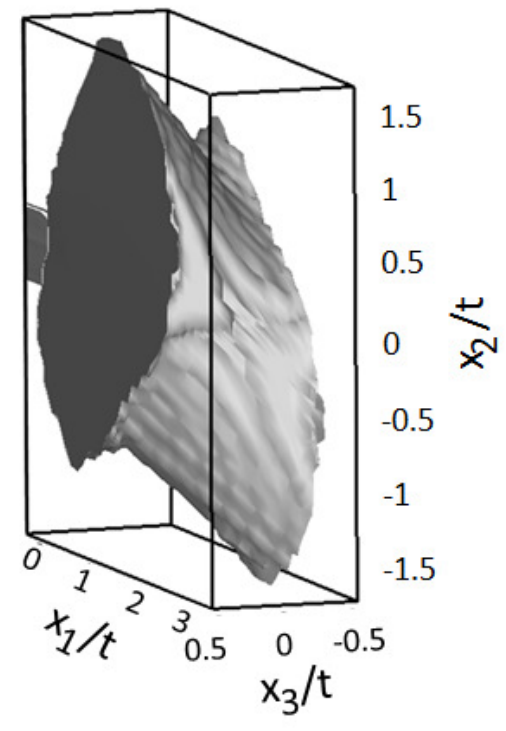

b)

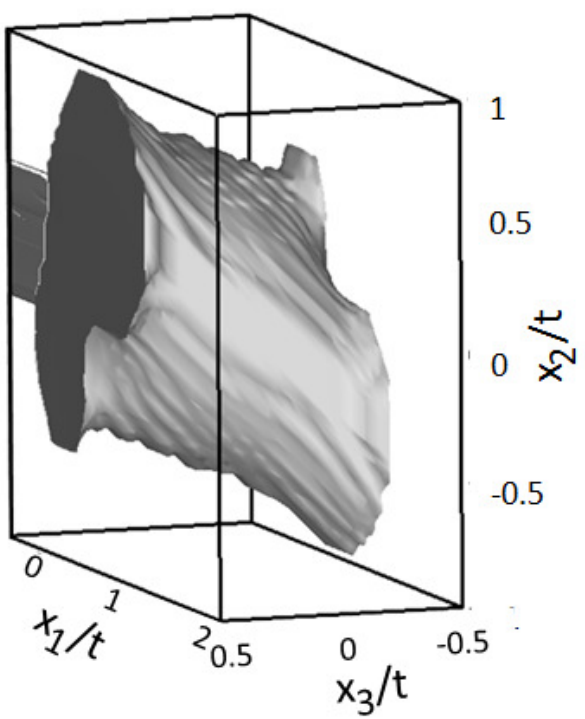

d)

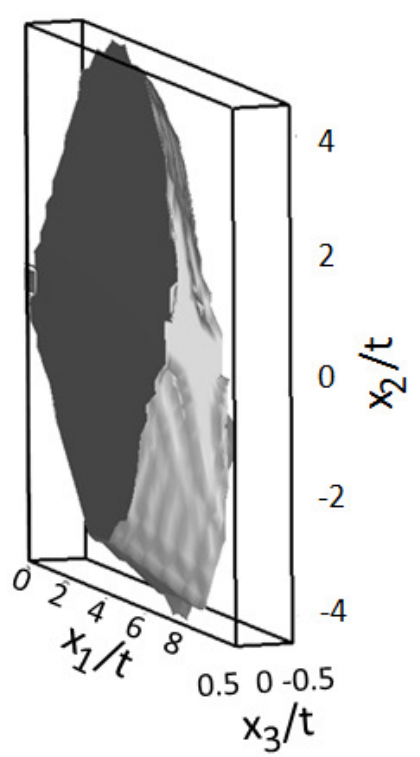

Fig. 10: Active plastic zone for a 45 degree slant crack propagating at steady-state with the loading corresponding to a) $K_{R} /\left(\sigma_{Y} \sqrt{t}\right)=1$, b) $K_{R} /\left(\sigma_{Y} \sqrt{t}\right)=2$, c) $K_{R} /\left(\sigma_{Y} \sqrt{t}\right)=3$, and d) $K_{R} /\left(\sigma_{Y} \sqrt{t}\right)=5$. Axes are normalized by the sheet thickness. 
a)
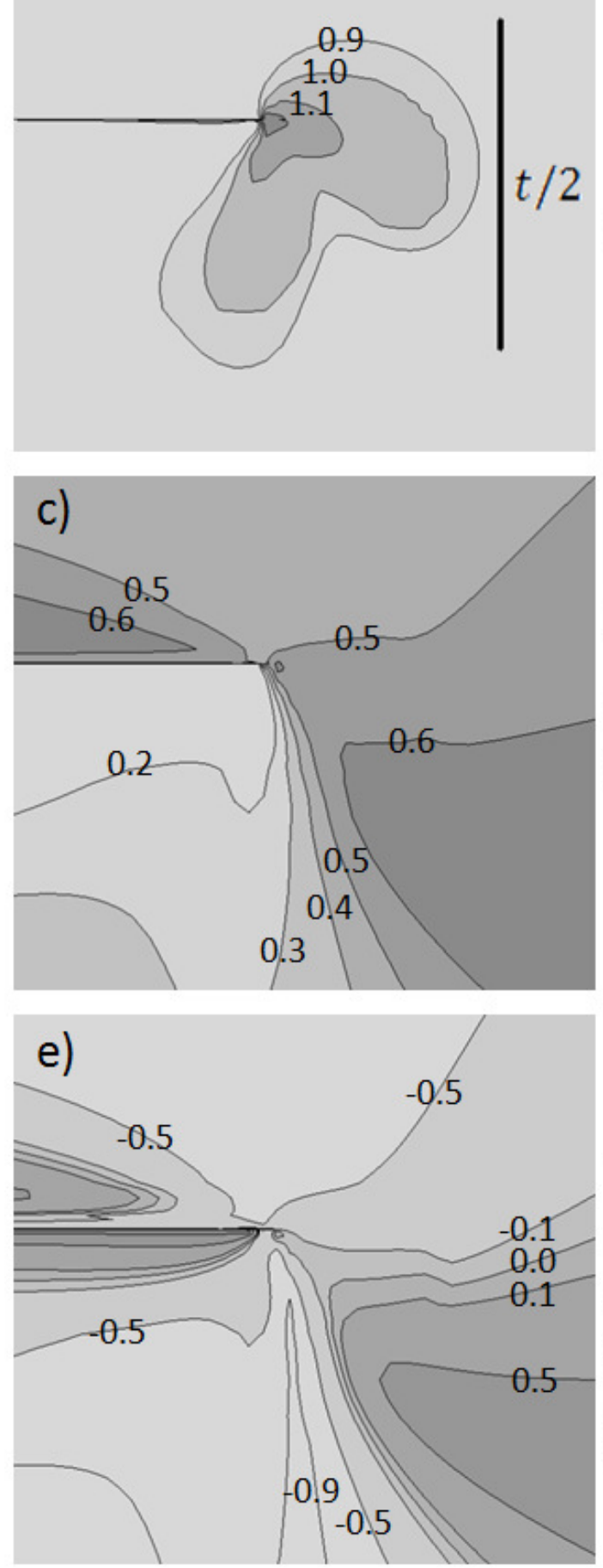

b) von Mises stress
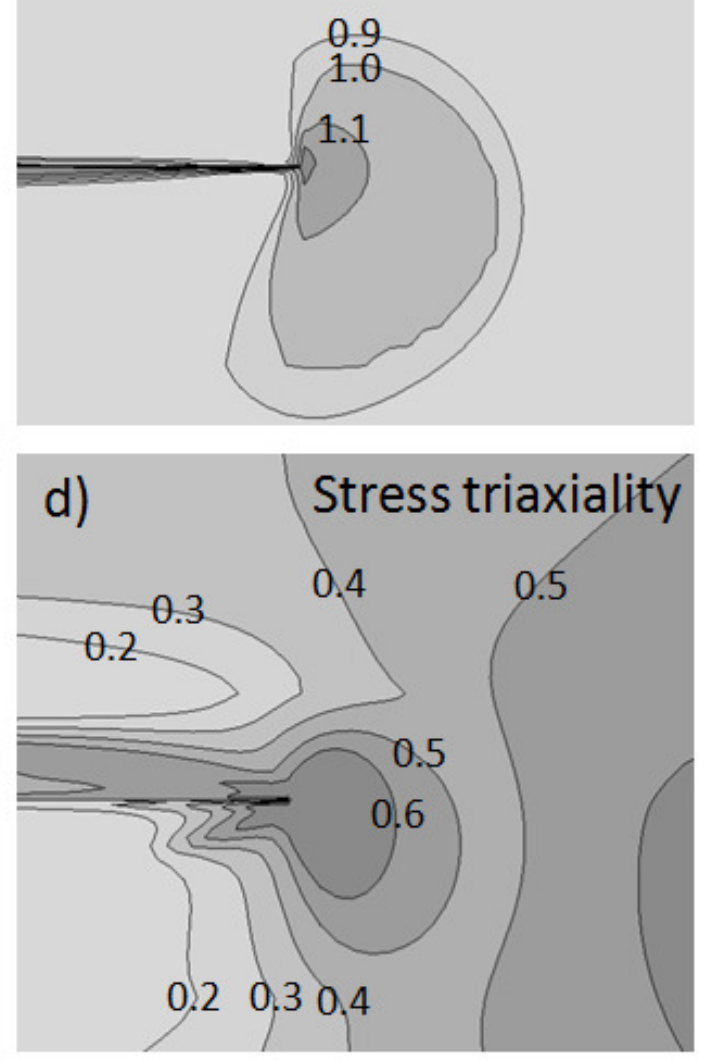

f)

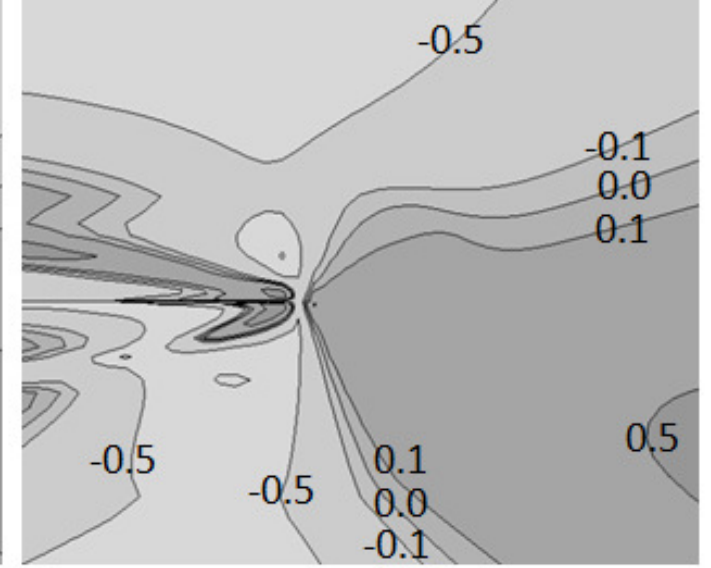

Fig. 11: Contour plot at $x_{3}=t / 2$ (free surface, plots; a, c, e) and at $x_{3}=t / 3$ (plots; b, d, f). Here, showing; a-b) normalized von Mises stress $\left(\sigma_{e} / \sigma_{y}\right)$, c-d) Stress triaxiality, and e-f) Lode parameter, $\left(K_{R} /\left(\sigma_{Y} \sqrt{t}\right)=1, t / H=0.01\right.$, and $\left.\beta_{S}=45^{\circ}\right)$. 

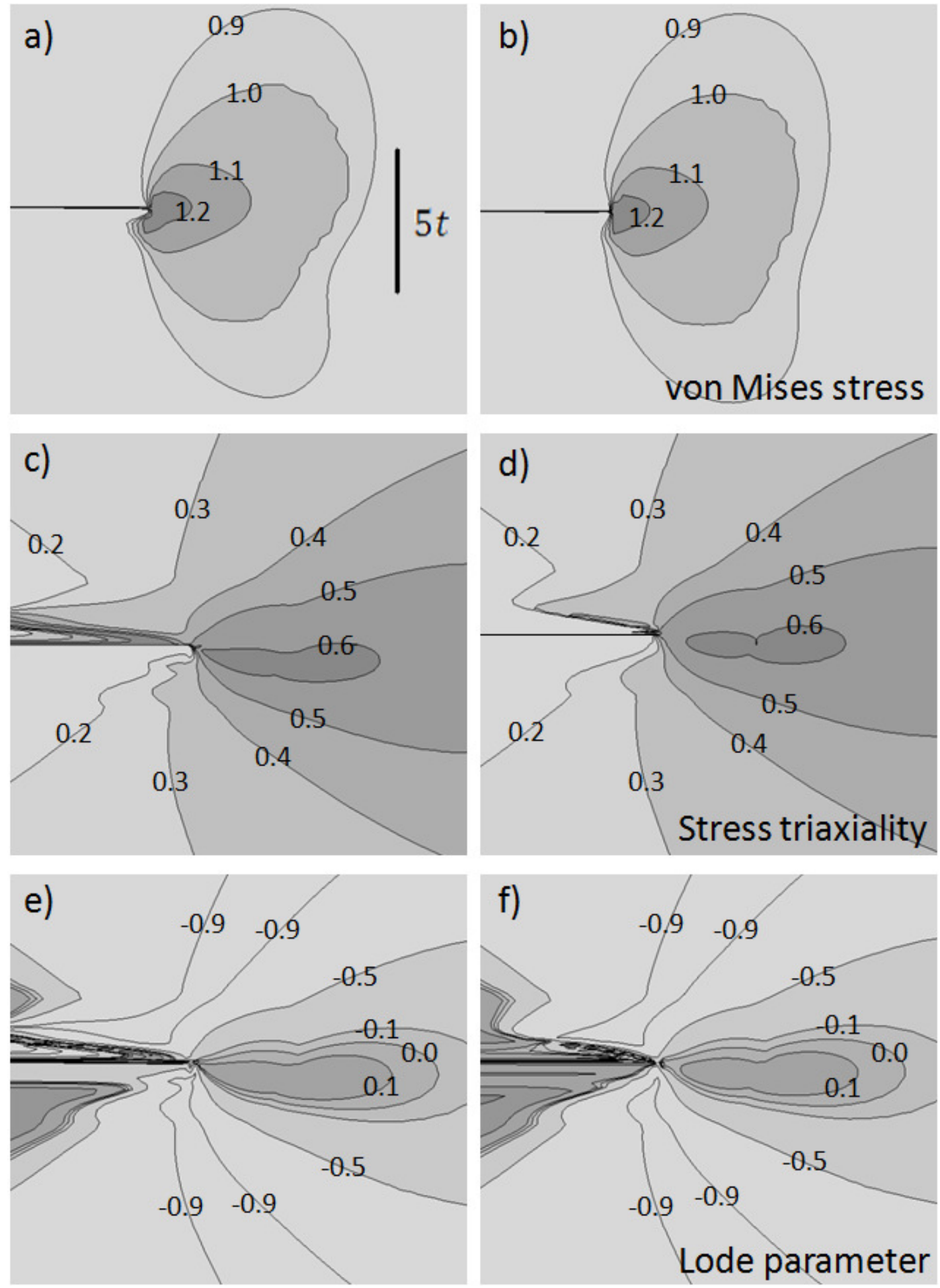

Fig. 12: Contour plot at $x_{3}=t / 2$ (free surface, plots; a, c, e) and at $x_{3}=t / 3$ (plots; b, d, f). Here, showing; a-b) normalized von Mises stress $\left(\sigma_{e} / \sigma_{y}\right)$, c-d) Stress triaxiality, and e-f) Lode parameter, $\left(K_{R} /\left(\sigma_{Y} \sqrt{t}\right)=5, t / H=0.01\right.$, and $\left.\beta_{S}=45^{\circ}\right)$. 


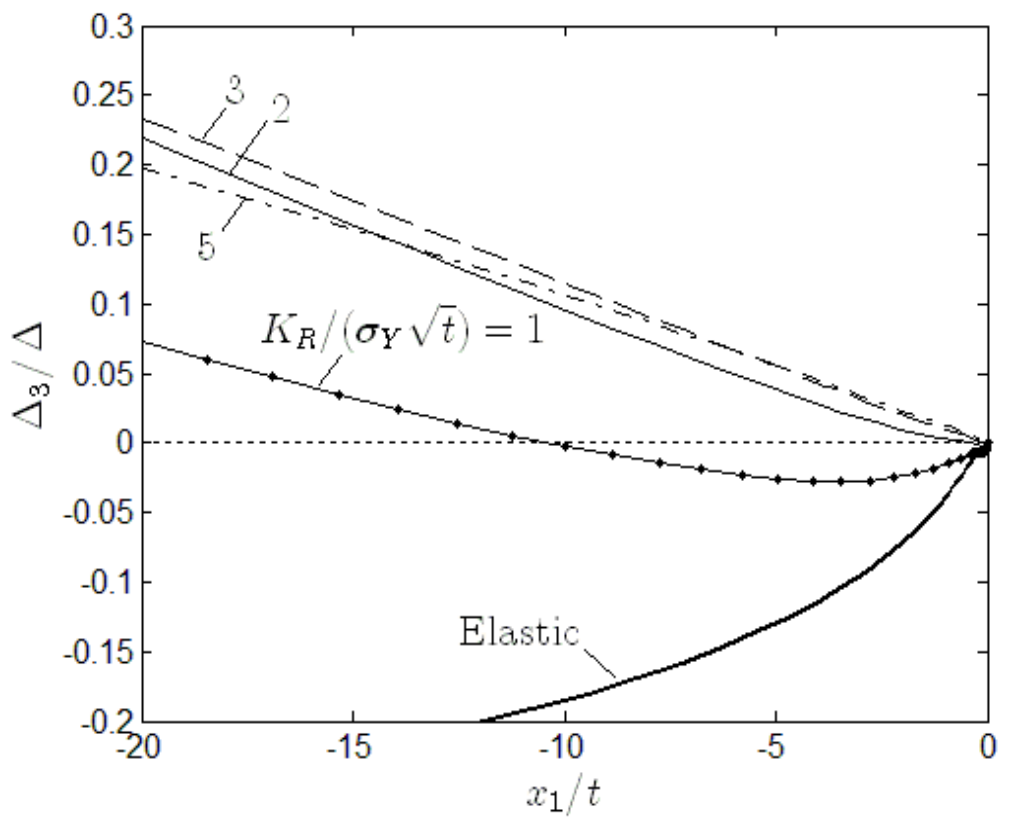

Fig. 13: Out-of-plane deflection for the elastic-plastic problem with $t / H=0.01$.

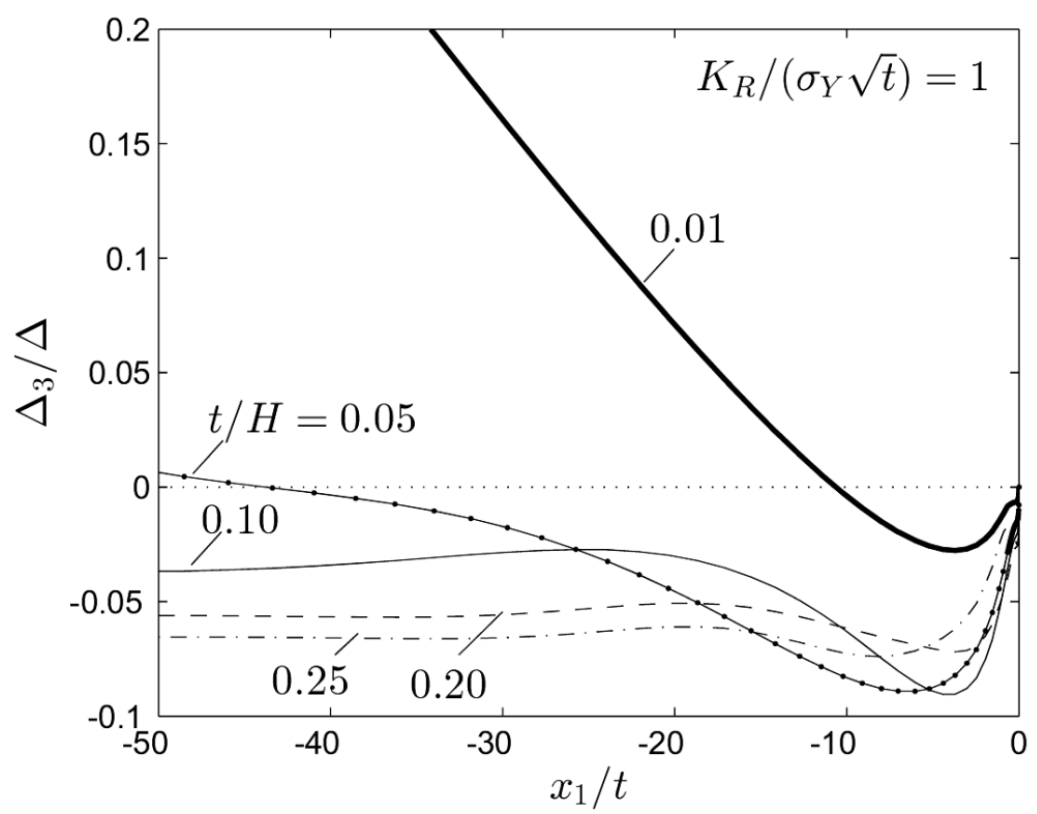

Fig. 14: Out-of-plane deflection for fixed $K_{R} /\left(\sigma_{Y} \sqrt{t}\right)=1$ and varying $t / H$. 